\title{
Análisis comparativo de dientes de rastrillo giratorio para uso agrícola
}

\author{
Comparative analysis of rotating rake teeth \\ for agricultural use
}

Claudio Roberto Acosta ${ }^{1}$, Jorge Alberto Halabí ${ }^{1}$

\footnotetext{
${ }^{1}$ Proyecto de Investigación en Análisis de Falla aplicado al Desarrollo de Componentes Regionales. Departamento de Ingeniería Mecánica, Facultad de Ingeniería, Universidad Católica de Córdoba, Av. Armada Argentina 3335, Córdoba, Córdoba, Argentina.

e-mail: materiales08ucc@gmail.com,jorgehalabi@ucc.edu.ar
}

\section{RESUMEN}

El presente estudio está alineado con el objetivo del proyecto de investigación para el apoyo a la pequeña empresa en el desarrollo de componentes regionales. Se presenta un caso de análisis realizado como asistencia a un productor en busca de la optimización en la calidad de su línea de uso agrícola. El solicitante del estudio requiere encontrar una causa raíz de la diferencia de comportamiento en servicio de los dientes de rastrillo lateral entre los de su propia fabricación y otros disponibles en el mercado local. Se trata de varillas de acero curvadas de diámetro $8 \mathrm{~mm}$ x $1300 \mathrm{~mm}$, las cuales se integran a ruedas multi-dientes que deben mostrar un comportamiento totalmente elástico durante su empleo.

El estudio se realiza sobre 4 muestras recibidas de diferentes productores.

Mediante análisis químico y metalográfico, medición de microdureza y ensayos de tracción, se han comparado las características de las diferentes muestras.

Se concluye que el tratamiento térmico de templado y revenido, posterior a la conformación en frío de la varilla, aumenta el límite de elasticidad en un promedio del $45 \%$ con respecto a las piezas que sólo tienen deformación plástica en frío. [3, 4, 5, 6, 7]

Palabras clave: diente, rastrillo, elasticidad, deformación en frío.

\section{ABSTRACT}

This study is aligned with the objective of the research project to support small business in the development of regional components. It is presented an analysis case performed as assistance to a producer in search of optimization in the quality of his line of agricultural use. The study applicant needs to find a root cause of the difference in in-service behavior of the lateral rake teeth between those of their own manufacture and others available on the local market. These are curved steel rods with a diameter of $8 \mathrm{~mm} \times 1300 \mathrm{~mm}$, which are integrated into multi-tooth wheels which must show a totally elastic behavior during their use.

The study is carried out on 4 samples received from different manufacturers.

Using chemical and metallographic analysis, microhardness measurement and tensile tests, the characteristics of the different samples have been compared.

It is concluded that the heat treatment of quenching and tempering, subsequent to the cold forming of the rod, increases the limit of elasticity by an average of $45 \%$ relative to the pieces that only have cold plastic deformation. [3, 4, 5, 6, 7]

Keywords: tooth, rake, elasticity, cold forming

\section{INTRODUCCIÓN}

En el presente trabajo, se realiza una evaluación comparativa de los diferentes grados de elasticidad y propiedades mecánicas, manifestados en varillas de acero de diámetro $8,00 \mathrm{~mm}$ pertenecientes a rastrillos giratorios de uso agrícola. Mientras algunos elementos sufren notables deformaciones permanentes en el funcionamien- 
to, otros mantienen su geometría mostrando mayor elasticidad ante el esfuerzo de flexión.

La falla detectada en servicio el uso se relaciona con una deformación permanente de las varillas que integran los dientes del rastrillo giratorio, lo que induce anomalías en el armado de la fila de producto cosechado y causando mermas en la recolección, afectando en forma negativa la productividad de la actividad. El estudio tiene como objetivo de identificar la causa raíz de la diferencia de comportamiento en el uso de cuatro muestras de dientes pertenecientes a distintos fabricantes.

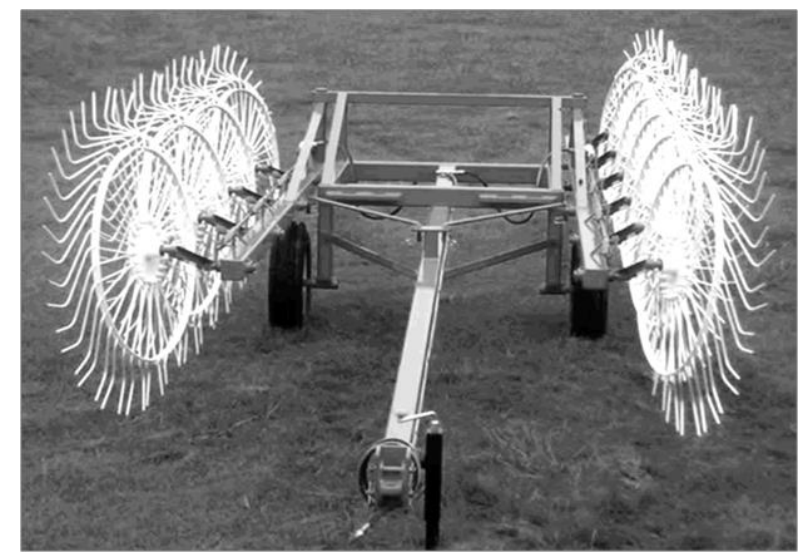

Figura 1: Implemento equipado con rastrillos giratorios

\section{MATERIALES Y MÉTODOS}

Mediante análisis químicos y metalográficos, medición de microdurezas y ensayos de tracción, se correlacionan los resultados obtenidos con la performance de los componentes en el servicio.

Los análisis químicos se realizaron mediante un espectrómetro de emisión modelo MAXxLMD05.

Los ensayos de tracción se realizaron con una máquina Instron 5.0T. [7]

En la observación metalográfica se empleó un microscopio Olympus mod. PME3.

La medición de durezas se realizó mediante un microdurómetro Leitz. [8]

\section{RESULTADOS}

\subsection{Análisis químico}

El análisis químico muestra que todas las piezas están construidas con el mismo tipo de material, un acero al carbono encuadrado en un SAE-1055.

\begin{tabular}{l|l|l|l|l}
\hline ELEMENTO QUIMICO & MUESTRA 1 & MUESTRA 2 & MUESTRA 3 & MUESTRA 4 \\
\hline $\mathrm{C}(\%$ en peso) & 0,54 & 0,54 & 0,55 & 0,54 \\
\hline $\mathrm{P}(\%$ en peso) & 0,011 & 0,013 & 0,013 & 0,013 \\
\hline $\mathrm{S}(\%$ en peso) & 0,014 & 0,014 & 0,014 & 0,013 \\
\hline Mn (\% en peso) & 0,63 & 0,65 & 0,63 & 0,65 \\
\hline Si (\% en peso) & 0.18 & 0.17 & 0,20 & 0.17 \\
\hline Cr (\% en peso) & 0,05 & 0,04 & 0,04 & 0,05 \\
\hline Ni (\% en peso) & 0,02 & 0,01 & 0,01 & 0,02 \\
\hline Mo (\% en peso) & $<0,01$ & $<0,01$ & $<0,01$ & $<0,01$ \\
\hline
\end{tabular}

Tabla 1: Análisis químico de las muestras. 


\subsection{Microdureza}

Los perfiles de microdureza se realizaron sobre un corte transversal de las varillas, con una trayectoria radial partiendo desde la superficie y hacia el núcleo. Se obtuvo lo mostrado en la tabla 1.

\begin{tabular}{l|l|l|l|l}
\hline $\begin{array}{l}\text { PROFUNDIDAD DE } \\
\text { PRUEBA }\end{array}$ & $\begin{array}{l}\text { MUESTRA 1 } \\
\text { HNV1 }\end{array}$ & $\begin{array}{l}\text { MUESTRA 2 } \\
\text { HNV1 }\end{array}$ & $\begin{array}{l}\text { MUESTRA 3 } \\
\text { HNV1 }\end{array}$ & $\begin{array}{l}\text { MUESTRA 4 } \\
\text { HNV1 }\end{array}$ \\
\hline $0,10 \mathrm{~mm}$ & 602 & 330 & 363 & 373 \\
\hline $0,30 \mathrm{~mm}$ & 642 & 353 & 353 & 353 \\
\hline $0,50 \mathrm{~mm}$ & 613 & 343 & 363 & 353 \\
\hline Núcleo & 624 & 363 & 384 & 363 \\
\hline
\end{tabular}

Tabla 2: Microdurezas sobre un corte transversal de las muestras. ASTM-384-16 [8] (promedio de 3 mediciones).

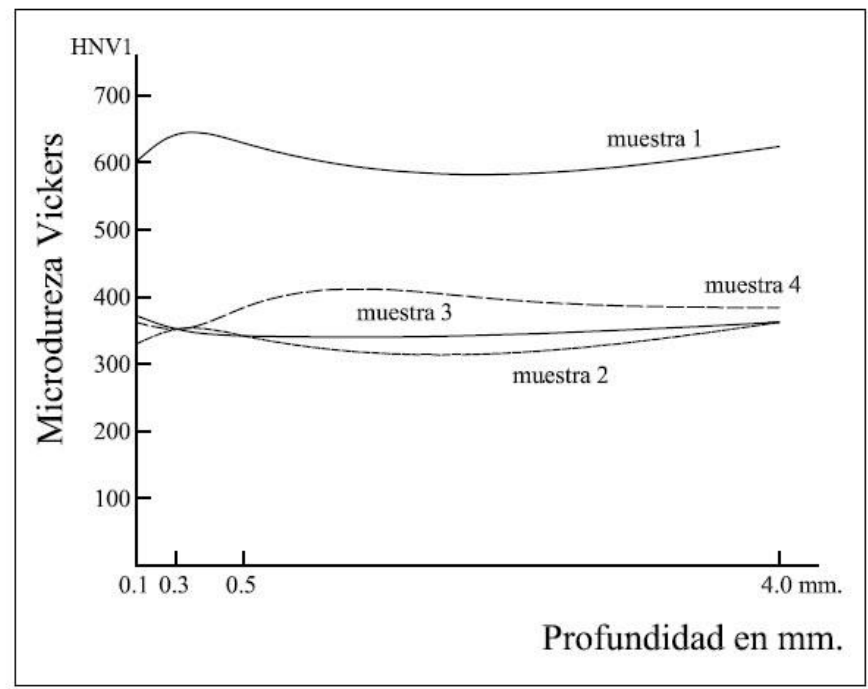

Figura 2: Perfiles de microdurezas en corte transversal de las muestras.

\subsection{Ensayo de Tracción}

Los ensayos se realizaron empleando probetas construidas en base a norma ASTM-A370-05 [7]. Los valores obtenidos se presentan en la tabla 3.

Tabla 3: Resultado de los ensayos de tracción de las muestras

\begin{tabular}{l|l|l|l|l}
\hline MUESTRA N $^{\circ}$ & $\mathbf{1}$ & $\mathbf{2}$ & $\mathbf{3}$ & $\mathbf{4}$ \\
\hline Tensión de Rotura (MPa) & 202 & 119 & 122 & 122 \\
\hline Tensión de Fluencia (MPa) & 170 & 107 & 111 & 110 \\
\hline Alargamiento (\%) & 3 & 4 & 6,8 & 6,8 \\
\hline Estricción (\%) & 24 & 54 & 58 & 54 \\
\hline
\end{tabular}




\subsection{Análisis Metalográfico}

Se realizaron cortes metalográficos en el sentido del eje principal de las varillas a fin de permitir la observación micro estructural y poner en evidencia posibles procesos de deformación plástica sobre las piezas estudiadas.
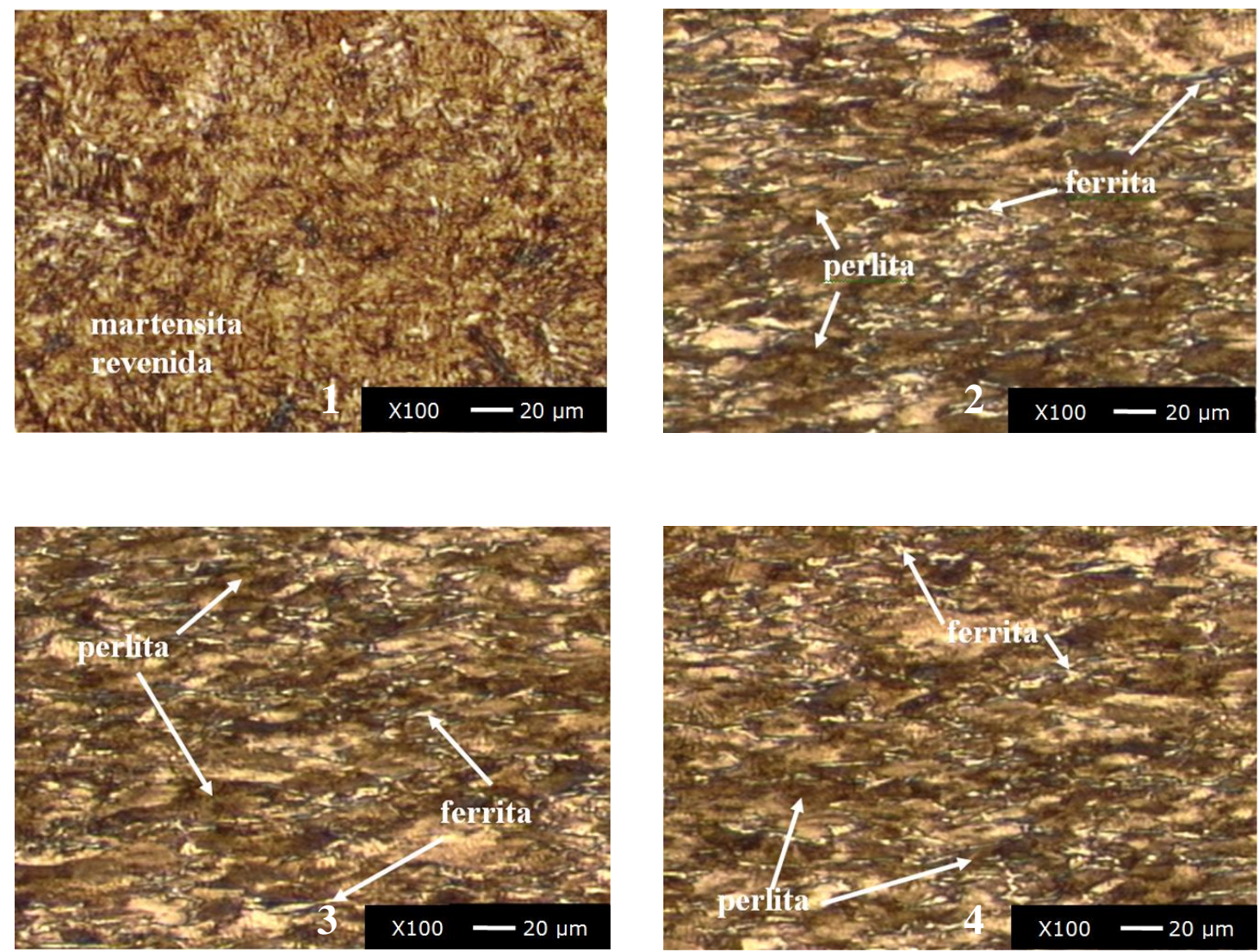

Figura 3: Microestructuras de muestras 1 a $4-\mathrm{x} 100$
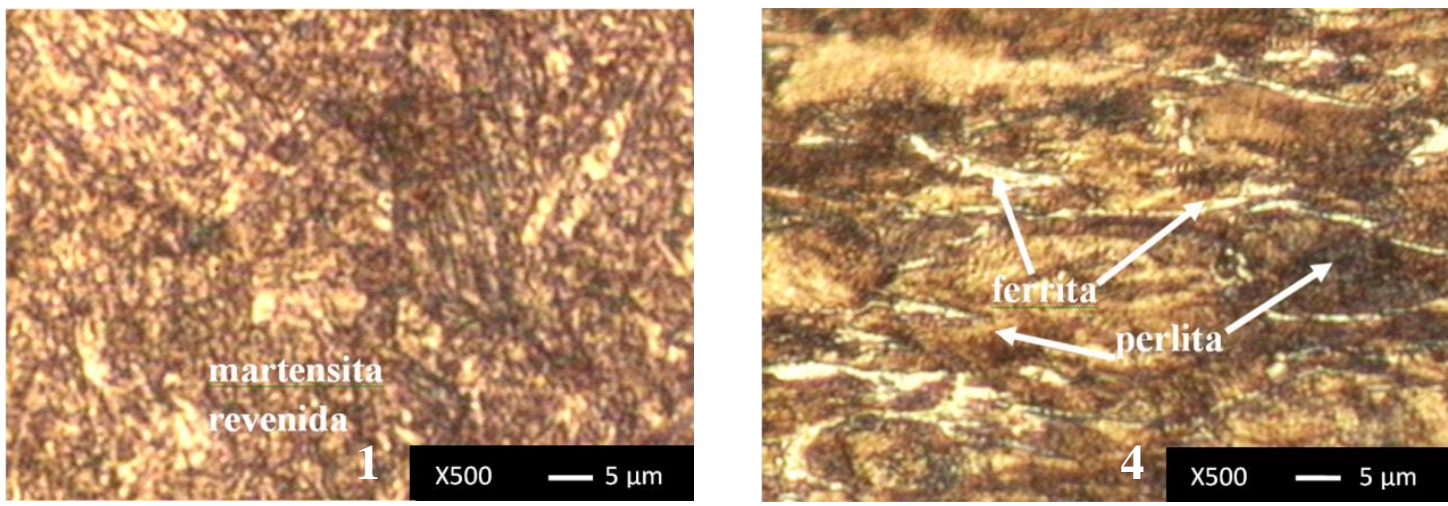

Figura 4: Microestructuras de muestras 1 y $4-$ x500

La microestructura de la muestra $\mathrm{N}^{\circ} 1$ está constituida por martensita revenida mientras que las tres muestras restantes presentan imágenes muy similares entre si y formadas por una predominancia de colonias perlíticas contorneadas por delgados canales de ferrita. 


\section{DISCUSIÓN}

La composición química evidencia que todas las muestras se encuadran dentro del mismo tipo de acero.

Las microdurezas, si bien difieren levemente entre las muestras 2, 3 y 4, éstas son significativamente diferentes a las que presenta la muestra $\mathrm{N}^{\circ} 1$, que posee durezas notoriamente mayores a las restantes tres muestras.

De manera similar a lo mostrado por las microdurezas medidas, los resultados de los ensayos de tracción evidencian una clara diferencia entre la muestra $\mathrm{N}^{\circ} 1$ y las restantes.

El examen metalográfico es coherente con las diferencias halladas en los anteriores ensayos ya que la muestra $\mathrm{N}^{\circ} 1$ (figuras 3 y 4 ) posee una microestructura de martensita revenida a diferencia de las tres restantes que están constituidas de ferrita y perlita laminar (figuras 3 y 4). En este último caso, hay evidencia de estiramiento de las colonias perlíticas y de los canales de ferrita, evidenciando que las tres muestras similares han sufrido un proceso de deformación plástica en frío [2]. La muestra $\mathrm{N}^{\circ} 1$ en cambio, ha recibido un tratamiento térmico de templado y revenido [4].

A causa de la forma geométrica filar de los dientes de rastrillo y su condición del trabajo, que debe adaptarse a las múltiples irregularidades del terreno, inducen a grandes deformaciones por flexión, las cuales originan tensiones elevadas que al superar en algunos casos el limite elástico del material, el componente deforma plásticamente, cambiando su forma original y disminuyendo así su capacidad operativa [1,3]. La muestra $\mathrm{N}^{\circ} 1$ en cambio, por ser poseer un límite elástico mayor, resulta ser la más apropiada.

\section{CONCLUSIONES}

1. El tratamiento de temple y revenido es la variable determinante de la mejora de comportamiento de la muestra $\mathrm{N}^{\circ} 1$ al otorgar un aumento en su límite de elasticidad del orden del $45 \%$.

2. Las muestras $\mathrm{N}^{\circ} 2,3$ y 4, endurecidas por trefilado en frío, poseen menor limite elástico y resistencia a la rotura que la $\mathrm{N}^{\circ} 1$.

3. Si bien el tratamiento térmico de temple y revenido representaría un costo adicional, el beneficio de mayor productividad y vida útil de la herramienta puede justificar la incorporación del mismo en el proceso productivo.

\section{AGRADECIMIENTOS}

Los autores agradecen a la Universidad Católica de Córdoba por el apoyo al grupo de investigación y a la empresa Sudosilo S.A. por facilitar la ejecución de parte de los ensayos en su Laboratorio.

\section{BIBLIOGRAFÍA}

[1] CANALE L., MESQUITA R., TOTTEN G., Failure Analysis of Heat Treated Steel Components, ASM. pp. 255-273

[2] SLOOF P. A., SCHSTER R. M., "Yield Strength Increase of Cold Formed Sections Due to Cold Work of Forming”, International Specialty Conference on Cold-Formed Steel Structures, Scholar'Mine, pp. 518 528,2000 .

[3] FAILURE ANALYSIS AND PREVENTION - Metals Handbook. ASM - v. 11. $9^{\mathrm{a}}$ ed. pp. 136 -144.

[4] "Endurecimiento del acero", REED HILL R.E. Principios de Metalúrgica Física -2ªed.cap.17, CECSA, 1971.

[5] HTUN M.S., KYAW S.T., LUIN K.T., Effect of Heat Treatment on Microstructures and Mechanical Properties of Spring Steel, Journal of Metals, Materials and Minerals, v. 18, n. 2, pp.191-197, 2008.

[6] SMITH W., HASHEMI J., Fundamentos de la Ciencia e Ingeniería de Materiales, McGraw-Hill, Cuarta Edición, pp 271-305, 2008.

[7] ASTM - A 370 - 05 - Standard Test Methods and Definitions for Mechanical Testing of Steel Products.

[8] ASTM- E 384-16 - Standard Test Method for Microindentation Hardness of Materials. 